\title{
Design and Implementation of Remotely- Monitored Single Phase Smart Energy Meter via Short Message Service (SMS)
}

\author{
D.A. Shomuyiwa \\ Momas Systems Nigeria Limited \\ Lagos, Nigeria
}

\author{
J.O. Ilevbare \\ Department of Electrical and Electronic Engineering, \\ Faculty of Technology, \\ University of Ibadan, Nigeria
}

\begin{abstract}
This paper focuses on the design and implementation of a single phase energy meter with remote monitoring capacity using shortmessage service (SMS). The energy meter was designed using ADE7755 metering chip and other discrete components. The SMS communication with the meter was established with the aid of Neoway M590 GSM module; AT-commands are sent to the GSM module from a mobile phone for communication. Also, a 4x3keypad was employed for loading energy tokens and obtaining energy information from the meter; the energy data (query) is then viewed on the liquid crystal display unit. The project was implemented on a locally- made single layer printed circuit board with the aid of Diptrace software. Simulation of the electronic circuit designs was carried out using Proteus software, 8051 development kit and a serial port monitor. Results obtained show that the energy meter is capable of measuring instantaneous and average real time power consumed while being remotely monitored and queried. The energy meter has a capacity of measuring minimum and maximum loads of $2 \mathrm{~mA}$ and $40 \mathrm{~A}$ respectively.
\end{abstract}

\section{Keywords:}

Energy meter, Remote Monitoring, Microcontroller, Tokens, Simulations, SMS

\section{INTRODUCTION}

Energy is an essential input to all aspects of human life [1]. It is employed for all levels of human activities. However, increased energy consumption and its rising cost per $\mathrm{kWh}$ informed the consumers of the need for effective energy utilization and monitoring [2]. Low energy consuming appliances such as Compact Fluorescent Lamps (CFL), Light Emitting Diodes (LED), etc, are being used for domestic and industrial illumination nowadays to reduce energy consumption costs. However, whether appliances possess low or high consumption capacity, it is necessary to monitor the quantity of energy consumed at any particular time. This is achieved by an energy metering system. Therefore, metering could be defined as the process of effectively determining and monitoring power consumption [3]. Prepaid metering requires the customer to make advance payment before electricity can be used while postpaid metering involves payment of energy consumed after usage.
In most developed nations, pre-paid metering is being employed on a full scale with remote monitoring capacity. Such energy metering technology is yet to be implemented on a large scale in most sub-saharan Africa region [4]. For example, since 1896 when the first power station was commissioned at Marina, Lagos, Nigeria, it has been observed that post-paid metering is ineffective due to unavailability of human resources, the manual methods of reading and storing data, human manipulations/errors and unavailability of the meters in rural areas [5]. The problems identified with manual metering system motivated the development of alternative methods (automatic) for determining the consumption of electricity. The primary aim of automation in meter reading is not only to reduce labor costs but also to resolve the problem of high system cost, inaccurate energy calculation, grounding and noise interfacing, tamper detection and fault clearance. In some cases, electricity meters are located in places that cannot be accessed by the utility company and require an appointment with the home owner for the purpose of meter reading. Electricity is a valuable commodity and the consumed energy should be measured through actual readings rather than estimated readings.

The conventional energy metering system has poor accuracy and lacks configurability; it also does not have means of detection when it is tampered with; however, recent research and industrial developments have provided opportunities in implementing energy efficient metering technologies that are more precise, accurate and error free [6]. Energy metering started with the conventional electromechanical meter whose mode of measurement is through a coil and rotating disc [7]. Advancement in technology brought about electronic meters with ability to measure using ADCs coupled with EPROM, RAMS and FLASH as storage devices. The only short comings of the electronic meter is the proprietary protocols which is resolved by smart meters with an open protocol such as DLMS protocol, and other capabilities such as remote connection/disconnection and PLC/GPRS/CDMA mode of remote communication [8].

In the United Kingdom, electronic token prepayment metering has been widely used for customers having poor record of payment [9]. A scholarly research work on Digital Tele-wattmeter System as an example of a microcontroller- based meter was presented by Shwehdi et.al [10]. The energy meter was implemented for the purpose of transmitting information about the energy consumed on a monthly basis to a remote central office through dedicated telephone line and a pair of modems [9]. Also, Zhang et al [11] employed a DSP-based meter in measuring energy consumption 
of multiple users in a residential area. A Personal Computer (PC) positioned at the control centre was used in sending commands to a remote meter, which in turn transmits data back [9], using the Power Line Communication (PLC) technique but the major shortcoming with this system is that it cannot detect tampering by consumers. A design which can be used for data transmission between the PC and smart card is proposed by Kwan et al [12]; data transmission from the personal computer to the smart card is possible via the smart card interface device. This has led to the development of an intelligent prepaid energy meter modeled and simulated by MATLAB/Simulink tools. Koay et al [13] presented the design and implementation of a bluetooth-enabled energy meter in which the user can get data on energy consumption from the meter through a wireless system. This system presents a challenge of distance coverage as bluetooth arrangement works effectively at close range.

The objective of this work is to design and implement a class 1 single phase meter (meter with a $1 \%$ tolerance) capable of measuring instantaneous and an aggregation of real time power consumed, which can be remotely monitored or queried. The remote monitoring is achieved through short message service. This design solves the challenge of taking readings manually from the meter, manual disconnection of meter and vending of tokens as a means of billing.

\section{METHODOLOGY}

\subsection{System Design}

The system designed has the following specifications: line voltage range of $180 \mathrm{~V}-250 \mathrm{~V}$, class 1 meter $( \pm 1 \%$ error) [14], starting current, $\mathrm{I}_{\mathrm{s}}=0.004 \mathrm{I}_{\mathrm{b}}, 3 \times 4 \mathrm{LCD}$ system, Neoway M590 GSM module, AT89C55WD Microcontroller programmed in $\mathrm{C}$ language-using Keil uVision3 compiler and Printed Circuit Board (PCB) designed with the aid of Diptrace Software. Figure 1 shows the components of the metering system.

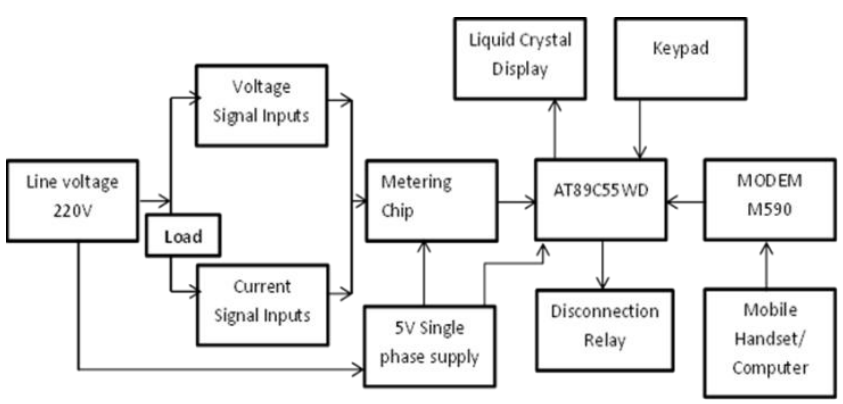

Figure 1: Components of the metering system

\subsection{The Energy Metering System}

The line voltage which is of the range of $180 \mathrm{~V}-220 \mathrm{~V}$ is fed into the volatge and current channels. This is essentially a network of resistors to attenuate the voltage to about $200 \mathrm{mV}$. The line voltage applied across the load produces the load current which is fed into the metering chip (ADE7755) for computation via current channel as shown in figure 2 [15]. The two ADCs digitize the voltage signals from the current and voltage transducers. These ADCs are 16-bit second order sigma-delta with an oversampling rate of $900 \mathrm{kHz}$. A programmable gain stage in the current channel further facilitates easy transducer interfacing. Also, a high-pass filter in the current channel removes any direct current (d.c) component from the current signal. This eliminates any inaccuracies in the real power calculation due to offsets in the voltage or current signals. The real power calculation is derived from the instantaneous power signal which was generated by a direct multiplication of current and voltage signals. In order to extract the real power component (i.e., the d.c component), the instantaneous power signal is low-pass filtered. This scheme correctly calculates real power for non-sinusoidal current and voltage waveforms at all power factors. All signal processing is carried out in the digital domain for superior stability over temperature and time [15]. The low-frequency output of the chip (ADE7755) is generated by accumulating the real power information. It indicates a long accumulation time between output pulses. The output frequency is therefore proportional to the average real power. The average real power information is accumulated by the MCU (AT89C55WD) to generate real energy information. Because of its high output frequency and shorter integration time, the output of the calibration frequency (CF) is proportional to the instantaneous real power. This is essential for system calibration purposes under steady load conditions [15].

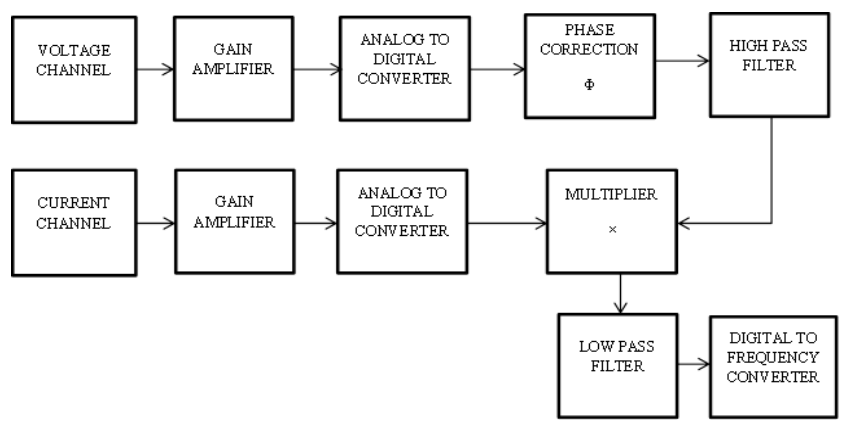

Figure 2: Functional block diagram of the chip [17] 


\subsection{Energy Meter Design}

The energy meter design has been sectioned into three parts (current channel, voltage channel and the ADE7752). The features have also been indicated in figure 3 . The voltage channel is a network of resistors used in attenuating the line voltage $(180 \mathrm{~V}$ $220 \mathrm{~V}$ ) to about $200 \mathrm{mV}$. The line voltage is applied across the load producing the load current which is fed to the metering chip (ADE7755) for computation through the current channel as shown in figure 3.The current channel is a fully differential input. The design is such that the maximum peak differential signal on the input should be less than $\pm 470 \mathrm{mV}$ (330mVrms for a pure sinusoidal signal) while the voltage signal on the input must be referenced to a common mode (ground).

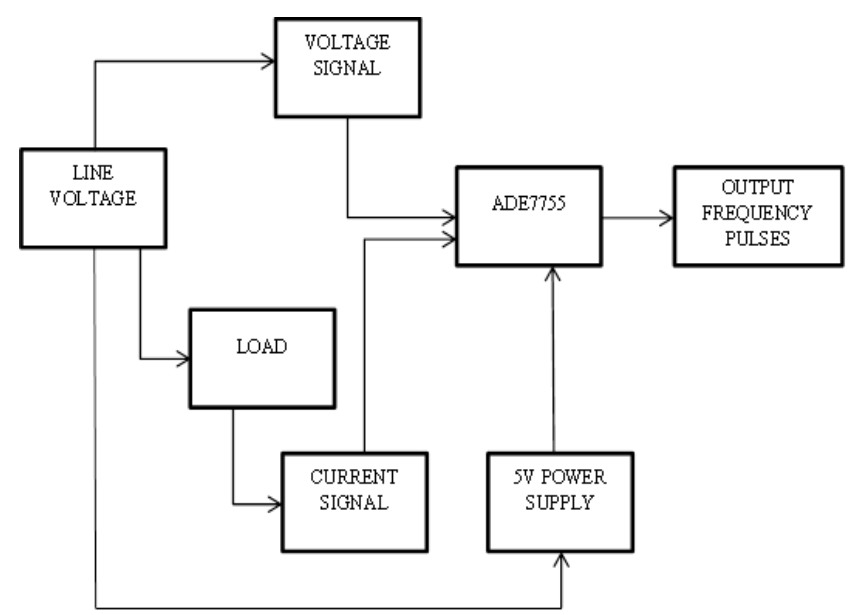

Figure 3: Block diagram of the energy meter design
The ADE7755 metering chip converts the measured voltage and current values from its input channels to their digital equivalents. The digital signals are sent to the multiplier from which the instanteneous power consumed is determined. Finally, the digitalto-frequency converter produces a train of pulses whose frequency is proportional to the computed value of power [16]. The calibration frequency which is the frequency output is connected to the MCU counter/port to count the number of pulses in a given integration time. This is determined by setting the MCU internal timer to 20 seconds. Equation 1 shows the relationship between the average power and the average frequency. Also, equation 2 represents the energy consumed during an integration period. Setting the integration time to 20 seconds will ensure accommodation of adequate pulses to achieve correct averaging of the frequency. The measured energy will still have ripples at this integration time but can be eliminated by setting the integration time to about 1 minute or more. Equation 3 is an expression for the expected output frequency.

Average frequency $=$ averagera real power $=$ counter/timer

Energy $=$ Average power $\times$ time $=$ counter $/$ time $\times$

time $=$ counter

frequency $=\left(8.06 \times V 1 \times\right.$ V2 $\times$ Gain $\left.\times F_{1-4}\right) /$ Vref $f^{2}$

where, $F_{\text {out }}=$ Output frequency

$(\mathrm{Hz})$,

$V 1=$ Differential rms voltage signal on Channel 1 (Volts) $=$ $470 \mathrm{mV}$

$V 2=$ Differential rms voltage signal on Channel 2 (Volts) $=$ $660 \mathrm{mV}$

Gain $=$ PGA gain selection made using logic inputs $\mathrm{G} 0$ and $\mathrm{G} 1=$ 1 (for $\mathrm{G} 0=\mathrm{G} 1=0)$ [17].

$V_{\text {ref }}=\quad$ Reference voltage $(2.5 \mathrm{~V} \pm 8 \%)$ (Volts)

$F_{1-4}=\quad$ One of four possible frequencies selected by using the logic inputs $\mathrm{S} 0$ and $\mathrm{S} 1=13.6$ (for $\mathrm{S} 0=\mathrm{S} 1=1$ ) [17]

$F_{\text {out }}=\frac{8.06 \times 0.47 \times 0.66 \times 1 \times 13.6}{(2.5)^{2}}$

$F_{\text {out }}=5.44 \mathrm{~Hz}$ 


\subsection{Interfacing the Keypad}

The keypad contains 12 keys arranged in four rows and three columns. The rows are connected to port 1 bits 3 to 6 while the column lines are connected to port 1 bits 0 to 2 . To detect a pressed key, the microcontroller grounds all rows by providing 0 to the output latch, and then read the data from the columns. If the data read from the columns is 111 , this means no key has been pressed. The process continues until a key press is detected. However, if one of the column bits has a zero, this means that a key press has occurred. After a key press is detected, the microcontroller will go through the process of identifying the key. Figure 4 shows the flowchart of the algorithm of the keypad interfaced with microcontroller. functioning. It is basically used to aid the communication of the meter through the microcontroller via SMS. It is a GSM/GPRS wireless module for dual band which includes SMS, data service and other functions. The module is used in two modes, either as SMS or GPRS but in this design it is switched to SMS mode. The modem comprises of a power supply, a voltage transition network and the module (Neoway M590). A 5V d.c supply goes into the regulator (MIC2930WU) in order to regulate the voltage to the required $3.5 \mathrm{~V}$ for the module at pin 2 and 3 of the microcontroller (AT89C55WD). The voltage transition network is a transistor network which acts as a buffer isolating the supply voltage from the $2.5 \mathrm{~V}$ expected at the output of the network. The SIM card provides a means of establishing communication via the GSM service provider and it is interfaced as shown in figure 5. Table 1 gives the description of SIM card interface.

\subsection{The Modem and SIM Card Interfacing}

The GSM module used is Neoway M590. The modem is described as a module and the circuit built around it for proper

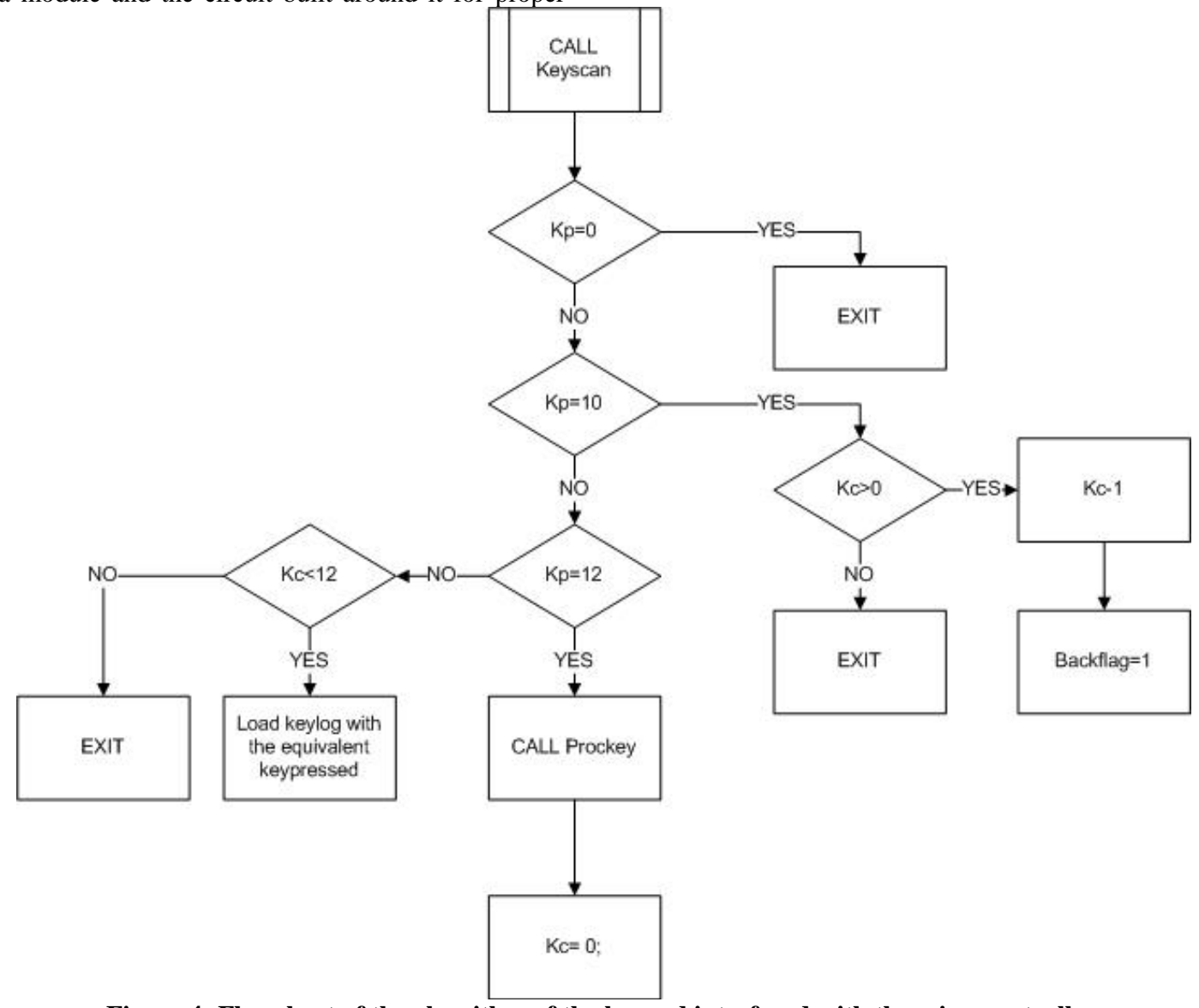

Figure 4: Flowchart of the algorithm of the keypad interfaced with the microcontroller 


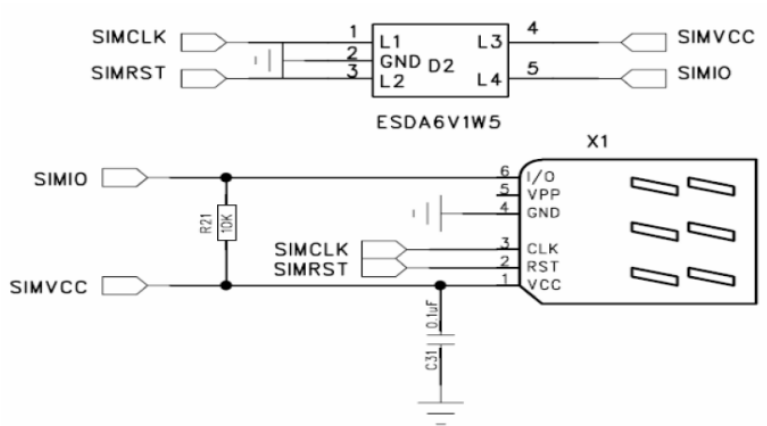

Figure 5: SIM card pin description (Shenzhen Neoway Technology Co. Limited, 2008)

Table 1: SIM card interface description [18]

\begin{tabular}{|c|c|c|c|l|}
\hline Pin & $\begin{array}{c}\text { Signal } \\
\text { name }\end{array}$ & I/O & $\begin{array}{c}\text { Function } \\
\text { Description }\end{array}$ & \multicolumn{1}{|c|}{ Remarks } \\
\hline 11 & SIMIO & I/O & $\begin{array}{c}\text { SIM card } \\
\text { data }\end{array}$ & $\begin{array}{l}\text { module external need } \\
\text { to connect pull up } \\
\text { resistance(10k) to SIM }\end{array}$ \\
\hline 12 & SIMCLK & O & $\begin{array}{c}\text { SIM card } \\
\text { clock }\end{array}$ & $\begin{array}{l}\text { Junction capacitance } \\
<20 \mathrm{pF}\end{array}$ \\
\hline 13 & SIMRST & O & $\begin{array}{c}\text { SIM card } \\
\text { reset }\end{array}$ & \\
\hline 14 & SIMVCC & PWR & $\begin{array}{c}\text { SIM card } \\
\text { power }\end{array}$ & \\
\hline
\end{tabular}

\subsection{Generation of Tokens and SMS Application}

The 16-digit energy tokens are codes generated using the software application program. It was developed using Delphi and C language. The energy tokens are given in the format $1 \$ \mathrm{xxx} . \mathrm{xx}$. The format is an encryption format by the software and tokens/codes loaded into the meter and also formatted in that order. The tokens are then loaded directly to the energy meter via the keypad. Also, the energy tokens can be loaded remotely via SMS sent from a mobile phone to the energy meter because the meter incorporates a modem and SIM card through which communication may be done. The text format for remotely loading the energy meter through SMS is done by sending a capital letter $\mathrm{T}$ followed by the 16-digit code. Apart from remotely loading the meter, the status: energy consumed and balances can be checked by sending a request via SMS. The user interface of token generation software is shown in figure 6 .
International Journal of Computer Applications (0975 - 8887)

Volume 74-No.9, July 2013

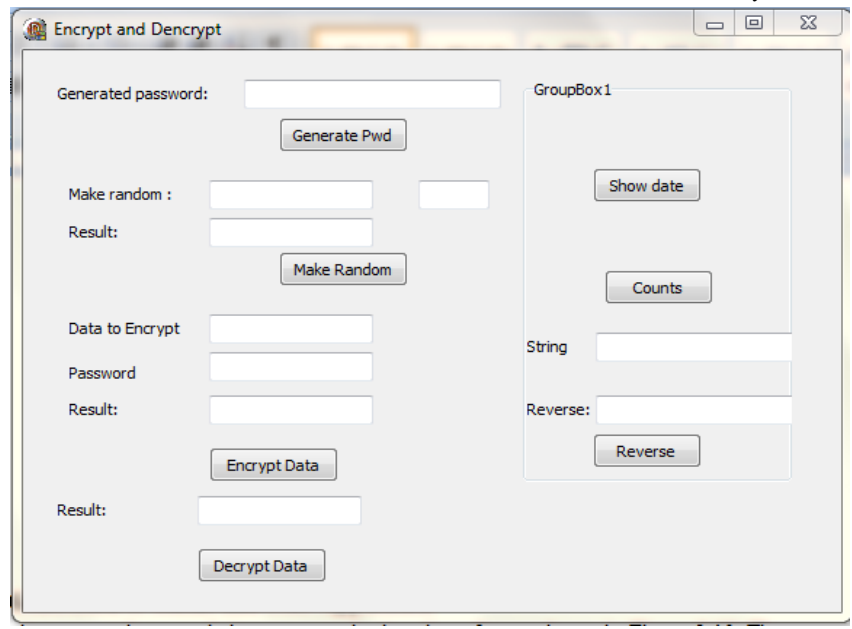

Figure 6: User interface of token generation software

\subsection{The Main Program}

The flow chart of the main program is shown in figure 7. It involves the initialization of all variables, calling of the display and keypad functions, checking the energy flag value, checking if the energy token has been exhausted and also freezing the data if exhausted until the meter is recharged. The program stores accumulated energy in Electrically Erasable Programmable ReadOnly Memory (EEPROM), deducts the energy tokens as it is being used and freezes the data when it has been exhausted. It also implements the sending of energy tokens to the meter via SMS from a mobile set via UART-9600 baud rate and gives a response back to the mobile set and a Real Time Clock (RTC) is also implemented. 


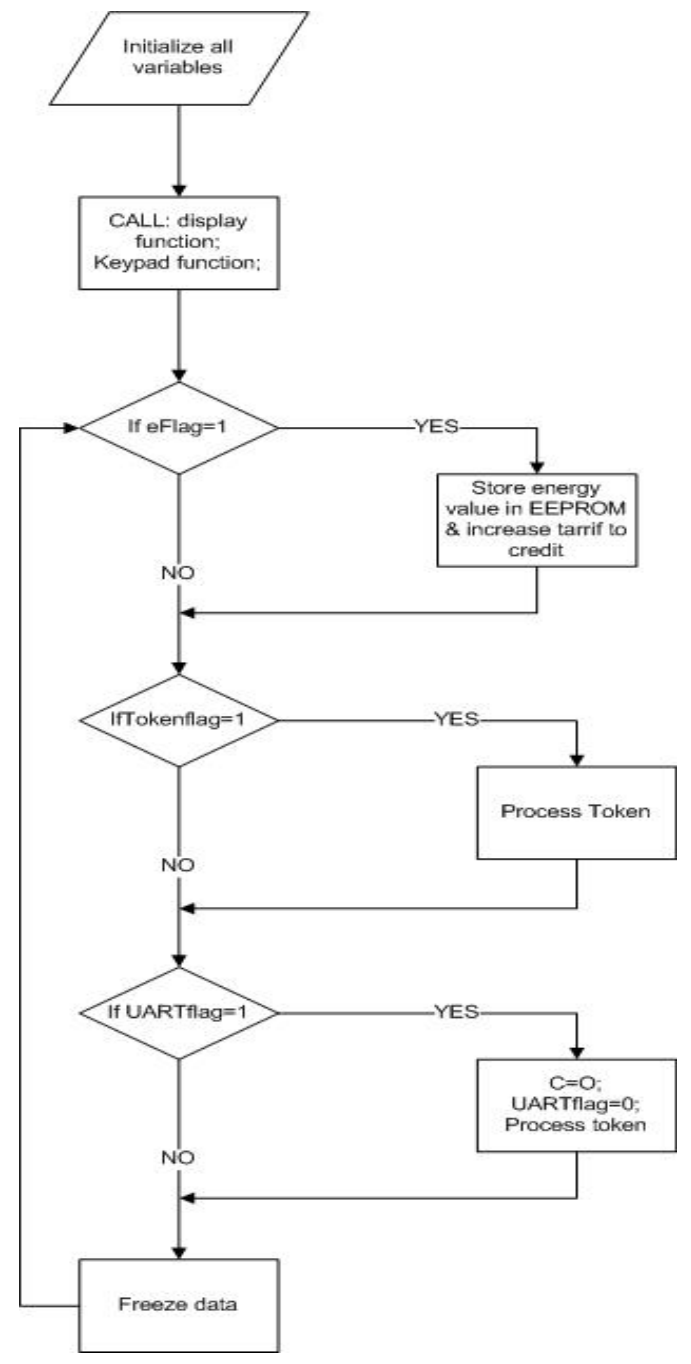

Figure 7: Flowchart of the main program

\subsection{PCB Design}

The Printed Circuit Board was designed with Novarm Diptrace version 2.1.0.7 software and printed on a transparent film paper. The foil protection on the ultra-violet copper clad board was removed, and the film paper side with the printing was placed on the board. It is then exposed to UV-exposure machine for about 90 minutes. The board was then dip exposed into a developer solution $\left(\mathrm{Na}_{2} \mathrm{CO}_{3}\right)$ and then shaken gently until prints develop. It was removed quickly from the solution when tracks were developed and then rinsed with water. The board was dried by tapping tissue on the surface and then further dipped in etching solution $\left(\mathrm{FeCl}_{2}\right)$ until the copper coating on the board leaves and thereby leaving only the tracks on the board. After this, the board was removed from the ferric solution, rinsed with water and then dried accordingly. Figure 8 shows energy metering circuit layout on the PCB and figure 9 shows the MCU circuit and modem layout.

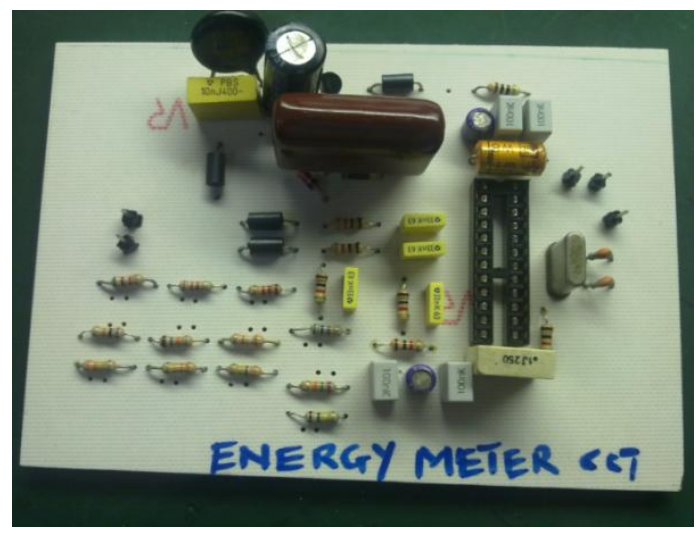

Figure 8: Single phase energy layout

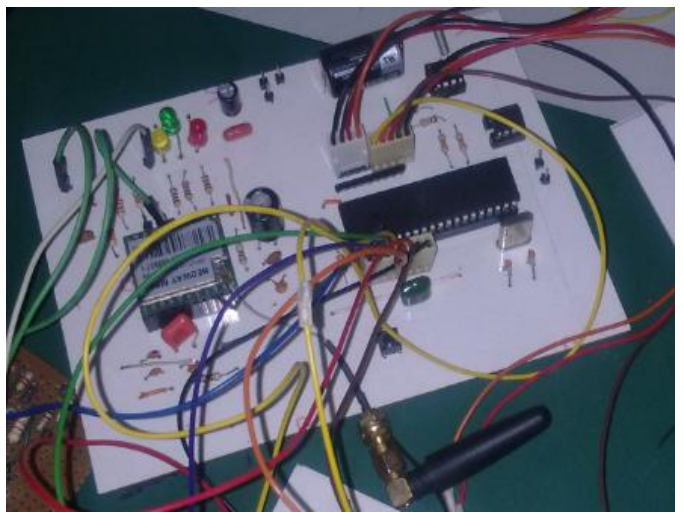

Figure 9: MCU and modem layout

\section{RESULT AND ANALYSIS}

The result has been separated into three sections namely: Simulation, Token generation and SMS application, and waveform and Test bench calibration result.

\subsection{Simulation}

The simulation of the project was done using the Proteus software and the 8051 development kit. The keypad, EEPROM, RTC and microcontroller were all tested in these environments. The keypad was used in loading energy tokens into the meter and also used to query the meter. The MCU, RTC, keypad and EEPROM were all successfully interfaced. Figure 10 shows the screen shot of the Proteus environment during the simulation. The Proteus environment is basically used to test the interfacing of the keypad and the ability of the RTC to retrieve real time clock and also to test the capability of the EEPROM to store accumulated energy data which were displayed via the LCD. 


\subsection{Tokens Generation and SMS Application}

\section{Test}

A 16-digit energy tokens was software generated, figure 6 shows the screen shot of the software. These tokens were punched through the keypad unto the meter to load the meter with the equivalent energy. Communication was also established by sending an SMS (to load energy tokens, check the energy balance and query the meter) via a mobile set as formatted by the encryption used in coding the token generation software to the meter by the GSM module and SIM card. A feedback response to the mobile handset for the requested query was received.

\subsection{Waveform and Test bench Calibration results}

Figures 11 and 12 respectively show the performance of the power supply unit under heavy load (50A) with line voltage varied between $180 \mathrm{~V}$ and $250 \mathrm{~V}$. This waveforms show stability of the power supply design under high and low voltage. Table 2 shows the Test bench results of the voltage-current readings while the power factor (both capacitive and inductive load) is being varied which indicates that the implemented meter is a class 1 type (meter design with an error of $\pm 1 \%$ ). Figure 13 shows the measured output waveform of the calibrated frequency as discussed in section 2.2.

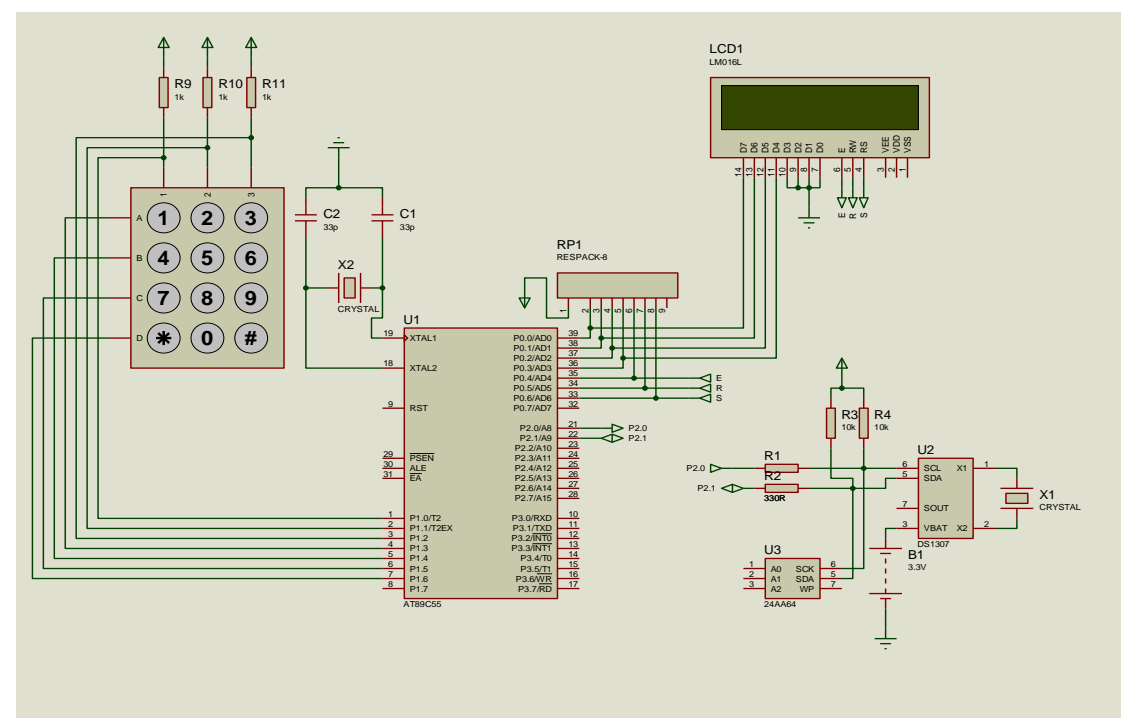

Figure 10: Layout of the schematic during simulation in Proteus environment

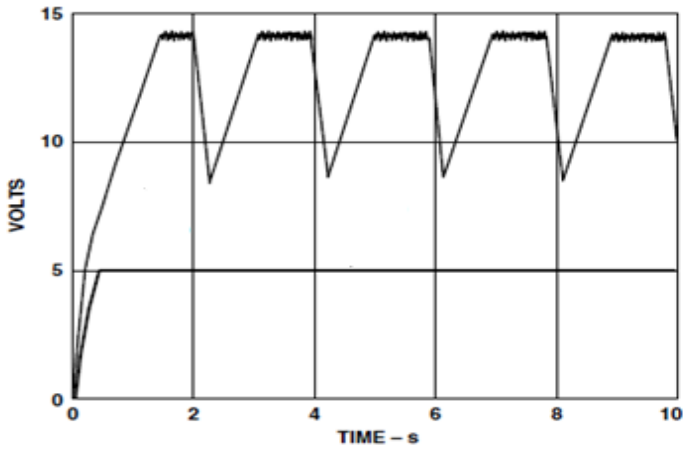

Figure 11: Power Supply output at $180 \mathrm{~V}$ and $40 \mathrm{~A}$

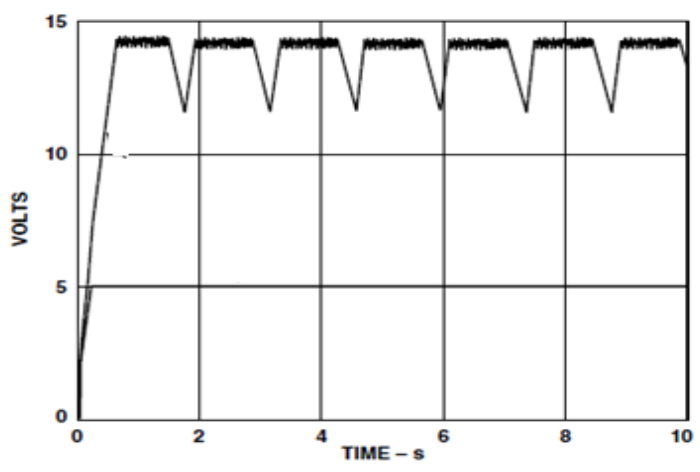

Figure 12: Power supply voltage output at $250 \mathrm{~V}$ and 40A 


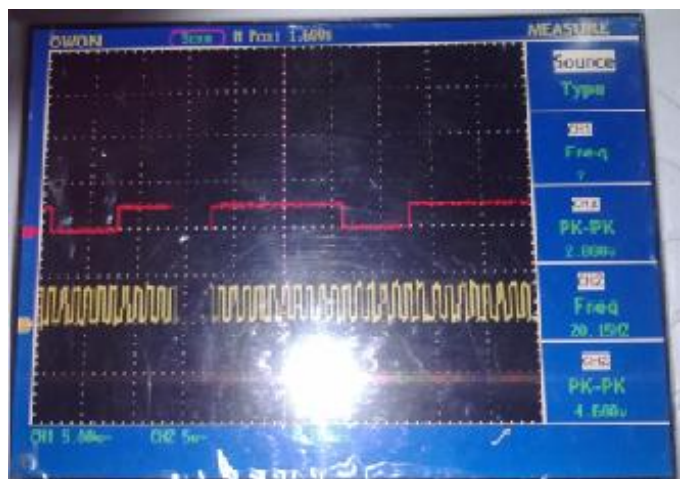

Volume 74-No.9, July 2013

Figure 13: Output waveform of the calibrated waveform

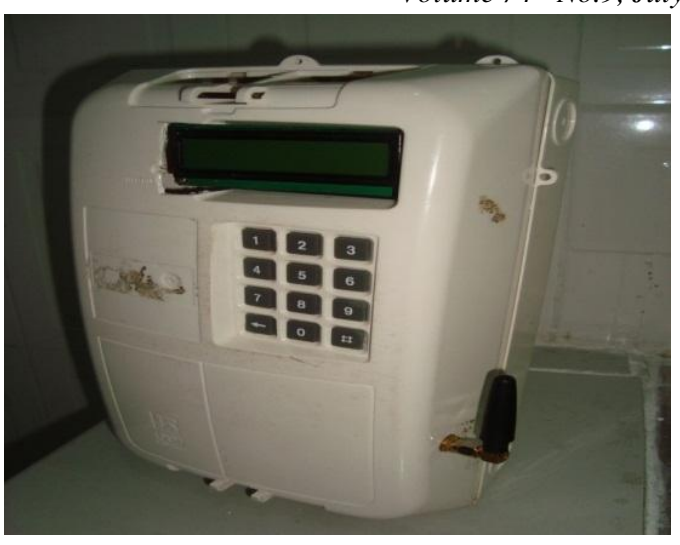

Figure 14: The packaged project

Table 2: Test bench results of the voltage-current readings with varying power factors

\begin{tabular}{|c|c|c|c|c|c|c|c|c|c|c|c|c|}
\hline \multirow{2}{*}{ Voltage(V) } & \multicolumn{4}{|c|}{$\mathrm{I}=30 \mathrm{~A}$} & \multicolumn{4}{|c|}{$\mathrm{I}=5 \mathrm{~A}$} & \multicolumn{4}{|c|}{$\mathrm{I}=0.25 \mathrm{~A}$} \\
\hline & $1 \mathrm{PF}$ & $0.8 \mathrm{C} \mathrm{PF}$ & $0.5 \mathrm{C} \mathrm{PF}$ & $0.5 \mathrm{~L}$ & $1 \mathrm{PF}$ & $0.8 \mathrm{C} \mathrm{PF}$ & $0.5 \mathrm{C} \mathrm{PF}$ & $0.5 \mathrm{~L} \mathrm{PF}$ & $1 \mathrm{PF}$ & $0.8 \mathrm{C} \mathrm{PF}$ & $0.5 \mathrm{C} \mathrm{PF}$ & $0.5 \mathrm{~L} \mathrm{PF}$ \\
\hline 264 & -0.1 & 0 & 0 & -0.1 & -0.1 & -0.1 & -0.12 & -0.3 & -0.1 & -0.2 & 0 & 0 \\
\hline 240 & -0.1 & 0 & 0 & -0.2 & -0.1 & -0.1 & 0 & -0.2 & -0.1 & 0 & 0.1 & -0.4 \\
\hline 144 & 0.63 & 0.2 & 0.1 & 0 & 0.22 & 0.2 & 0.1 & 0.46 & 0.47 & 0.24 & 0.1 & 0.3 \\
\hline
\end{tabular}




\subsection{Packaging}

The energy board, keypad, display and the modem and microcontroller boards are all integrated in a white plastic casing for compatibility. A Conlog meter casing was used to package the meter. The plastic casing has the LCD showing through it. The casing package has 2 input and output slots each all passed through the terminal block. Figure 14 shows the meter packaged in the Conlog casing.

\section{CONCLUSION}

Advancement in technology has provided means of effectively measuring and monitoring energy. Such technology has been achieved in this work by employing a modem and SIM card interface. A single phase energy meter with remote monitoring and energy token generation capability using SMS has been designed, modeled and constructed. The entire design has been implemented on a locally made single layer PCB. This design will address energy metering problem and help improve the billing systems with added advantage of remotely monitoring energy usage.

\section{ACKNOWLEDGEMENT}

The authors acknowledged the contributions and support of the following individuals to this work; Mr. A. O. Okike, Prof A. Olatunbosun, Department of Electrical and Electronic Engineering, University of Ibadan, and Daniel Akinyele, School of Engineering and Computer Science, Victoria University of Wellington.

\section{REFERENCES}

[1] Sambo AS, Renewable Energy for Rural Development: The Nigerian Perspective. ISESCO Science and Technology Vision 2005;1: 12-22.

[2] Bulbenkiene V, Pecko A, Zulkas E, Kuprinavicius A, Sokolov A and Mumgaudis G, Energy Sub-Metering Data Acquisition System. Electronics and Electrical Engineering 2011; 5(111): 99-102

[3] Balakrishna, 2009. World Meter Design Corporation. [Online] Freescale semiconductor Productions Available at: http://freescale.com [Accessed 8 August 2011].

[4] Nwaoyo I, Design and Construction of a Smart Card Based Single Phase Meter. Final Year Project. Department of Electrical and Information Engineering, Covenant University 2011.

[5] Lanphur C, Electric Meter history and Progress. Handbook. Westinghouse Press 1925.
International Journal of Computer Applications (0975 - 8887)

Volume 74- No.9, July 2013

[6] Mejbaul H, Kamal H, Mortuza A and Rafiqul IS, Microcontroller based Single Phase Digital Prepaid Energy Meter for Improved Metering and Billing System. International Journal of Power Electronics and Drive System 2011; 1(2): 139-147

[7] Falcone, 2002. MultiPhase Flow Metering: Current Trends and Future Development. Society of Petroleum Engineers, 54, pp.77-84.

[8] Chengi, 2008. [Online] Kalkitech Intelligent Energy System Productions (2.3) Available at: http://kalkicommunications.com [Accessed 26 August 2011].

[9] Omijeh BO and Ighalo GI, Design and Simulation of Single Phase Intelligent Prepaid Energy Meter. Innovative Systems Design and Engineering 2013; 4(1).

[10] Shwehdi MH and Jackson C, A Microprocessor Based Digital Wattmeter System Design. Proceedings of the $31^{\text {st }}$ Intersociety Conference on Energy Conversion Engineering 1996; 31:1840-1845

[11] Zhang JO and Bai CL, DSP Based Electricity Meter With Remote Reading. Proceedings of the $4^{\text {th }}$ International Conference on Signal Processing 1998; 2:1581-1584.

[12] Kwan BH and Moghavvemi M, PIC-Based Smart Card Prepayment System. Student Conference on Research and Development 2002; 440:443.

[13] Koay BS, Cheah SS, Sng YH, Chong PHJ, Shun P and Tong YC, Design and Implementation of a Bluetooth Energy Meter. Proceedings of the Joint $4^{\text {th }}$ International Conference on Information, Communication and Signal Processing and the $4^{\text {th }}$ Rim Conference on Multimedia 2003; 3:1474-1477.

[14] ISO/IEC, 2006. International Standard Organization. 3rd ed. IEC press.

[15] Analog Devices Inc., 2002. Energy Metering Ic with Pulse Output. Data Sheet, 13 January, pp. 1-16.

[16] Vector Institute, Vector's Advanced Embedded Development Board for 8051 User Manual 2010. Hyderabad: Vector Institute Press Vector Institute.

[17] Collins A, A Low Cost Watt Hour Energy Meter Based on the ADE7755. Application note 1999: 2-20.

[18] Okeola, M., 2008. GPRS Meter Technical Manual. Manual. Lagos: Momas Systems Nigeria Limited.

[19] Novarm, 2008. Diptrace Corporation. [Online] (2.3) Available at: http://www.diptrace.com [Accessed 25 March 2011]. 